A. G. Coran

\title{
Interleukin-6 changes tight junction permeability and intracellular phospholipid contentin a human enterocyte cell culture model
}

Accepted: 10 December 2002/Published online: 6 May 2003

(C) Springer-Verlag 2003

\begin{abstract}
Proinflammatory cytokines and secretory phospholipase $\mathrm{A}_{2}\left(\mathrm{sPLA}_{2}\right)$ are elevated in patients with inflammatory bowel disease (IBD). We previously reported that the proinflammatory cytokine IL-6 increased the expression of $\mathrm{sPLA}_{2}$ (a hydrolyzer of phosphatidylcholine) and decreased membrane integrity in an intestinal epithelial cell culture model. To determine the physiological effects of the IL-6 mediated increase in $\mathrm{SPLA}_{2}$ on decreased epithelial layer integrity, we investigated alterations of intracellular/secretory phospholipid (PL) composition in a cell culture model. In addition, since other PLs may also mediate epithelial membrane activity, we investigated the effect of IL-6 on PL activity in a Caco-2 enterocyte culture model. Caco-2 cells were incubated for $72 \mathrm{~h}$ with IL-6 or media alone (control). Both media and cell lysate were analyzed for PL composition using thin-layer chromatography. The PL composition in the media did not show any differences between the two groups $(p>0.1)$. Total intracellular PL contents were also unchanged; however, IL-6 led to significant changes in PL composition including an increase in phosphatidylethanolamine (PE) and sphingomyelin (SM) and a decrease in phosphatidylcholine (PC) and lysophosphatidylcholine (LPC) $(p<0.05)$. Both PE and SM are known as inflammatory signaling factors involved in human IBD. Our study suggests that the decreased membrane integrity seen with IL-6 application may occur via intracellular PL alterations, rather than through the direct effects of $\mathrm{sPLA}_{2}$.
\end{abstract}

Y. Tazuke · R. A. Drongowski · D. H. Teitelbaum

A. G. Coran $(\bowtie)$

Section of Pediatric Surgery,

University of Michigan Medical School,

F3970 Mott Children's Hospital, Ann Arbor,

Michigan, 48109-0245, USA

E-mail: acoran@umich.edu

Fax: + 1-734-9369784
Keywords Proinflammatory cytokines · Interleukin-6 • Secretory phospholipase $A_{2} \cdot$ Phospholipid $\cdot$ Epithelial permeability $\cdot$ Inflammatory bowel disease

\section{Introduction}

Elevated proinflammatory cytokine levels are present in patients with inflammatory bowel disease (IBD) $[1,2,3]$. In particular, the stimulated inflammatory cytokine interleukin-6 (IL-6) seems to play an important role in maintenance of immune suppression in septic patients $[4,5]$. In a human intestinal epithelial cell culture model, IL- 6 has been shown to mediate a selective down-regulation of enterocyte membrane hydrolase, which normally protects the intestinal barrier function [6, 7]. Another mediator elevated in septic shock is secretory phospholipase $\mathrm{A}_{2}\left(\mathrm{sPLA}_{2}\right)$, which is a major enzyme produced in response to proinflammatory cytokines and is found in inflammatory fluids as well as in the serum of patients suffering from septic shock $[8,9]$.

The presence of $\mathrm{SPLA}_{2}$ may have significant physiological influence. It has been reported that the $\mathrm{PLA}_{2}$ hydrolysis product of phosphatidylcholine (PC), lysophosphatidylcholine (L-PC), causes a loss of epithelial barrier integrity in both rat models $[10,11]$ and an enterocyte cell culture model [12]. We have previously shown that the proinflammatory cytokine IL-6 increases $\mathrm{SPLA}_{2}$ concentrations and decreases cellular membrane integrity in an intestinal epithelial cell culture model (presented at the 14th International Symposium on Pediatric Surgical Research, 2001, Madrid); however, the specific physiological effect of increased $\mathrm{SPLA}_{2}$ secretion in this model is unknown. To determine the physiological effects of the IL-6 mediated increase in $\mathrm{sPLA}_{2}$ on decreased epithelial layer integrity, we investigated alterations of intracellular/secretory phospholipid (PL) composition in this cell culture model. 


\section{Materials and methods}

Cell culture

Human enterocyte Caco-2 cell line (no. HTB37) were obtained from the American Type Culture Collection (Manassas, Va.). Caco-2 cell line passages 26-35 were grown in Dulbecco's modified Eagle's medium (DMEM), supplemented with $10 \%$ fetal bovine serum (Fisher, Pittsburgh, Pa.), 1\% non-essential amino acids (Gibco, Grand Island, NY), 1\% sodium pyruvate (Fisher, Pittsburgh, Pa.), penicillin $\mathrm{G}(100 \mathrm{U} / \mathrm{ml})$, and streptomycin $(100 \mathrm{~g} / \mathrm{ml}$; Fisher, Pittsburgh, Pa.), in a $5 \% \mathrm{CO}_{2}$ atmosphere at $37^{\circ} \mathrm{C}$. After reaching $60-70 \%$ confluence, cells were harvested by trypsinization with trypsin-EDTA (Gibco, Grand Island, N.Y.), washed and resuspended in DMEM. Caco-2 cells were seeded onto two types of cell culture plates at a density of $1 \times 10^{5}$ cells per well; one was a $3-\mathrm{cm}$ diameter cell culture dish and the other Transwell cell culture inserts with $0.33-\mathrm{cm}^{2}$-sized porous filters (pore size $0.3 \mu \mathrm{m}$ ), coated with $1.0 \mathrm{mg} / \mathrm{ml}$ rat-tail type-I collagen (Sigma, St. Louis, Mo.) in a two-chamber cell culture system (Costar, Cambridge, Mass.). Caco-2 cells were then grown for 14 days in media to allow them to reach confluence and fully differentiate. Media were changed every second day. Cell monolayers were used after a TEER of over $200 \mathrm{ohm} / \mathrm{cm}^{2}$ was measured in the $0.33-\mathrm{cm}^{2}$-sized filters.

\section{Experimental models}

Well-differentiated Caco- 2 cell monolayers in Transwell cell culture systems were used for investigating the effect of cytokine IL-6 (US Biological, www.usbio.net) on epithelial integrity by measuring transepithelial electrical resistance (TEER) and mannitol paracellular permeability. The cell monolayers were then incubated with media or media with the cytokine IL-6 $(10 \mathrm{ng} / \mathrm{ml})$ in the basal chamber for $72 \mathrm{~h}$.

Transepithelial electrical resistance (TEER) was measured before and $72 \mathrm{~h}$ after cytokine treatment using an epithelial volt ohmmeter (EVOM; World Precision Instruments, Sarasota, Fla.). The TEER values obtained in the absence of cells were used as background, and resistance was expressed as ohm $\times$ square centimeters $\left(\mathrm{ohm} \mathrm{cm}^{2}\right)$. Media samples from the apical and basal chambers were then collected for total $\mathrm{sLA}_{2}$ activity and phospholipid concentration.

${ }^{3} \mathrm{H}-\mathrm{Mannitol}$ was used as another measure of cellular monolayer integrity. After $72 \mathrm{~h}$ incubation with media, or media with IL-6, the monolayers were washed with media and then ${ }^{3} \mathrm{H}$-mannitol (molecular formula weight 182.2) was administrated into the apical chamber for investigating permeability. After $2 \mathrm{~h}$ incubation with ${ }^{3} \mathrm{H}$-mannitol at room temperature or at $4{ }^{\circ} \mathrm{C}$, the media in the basal chamber was collected and measured for the presence of ${ }^{3} \mathrm{H}$ mannitol. The data are expressed as percent ratio of tritium in the basal chamber / (tritium in basal chamber + the tritium in apical chamber $\times 100$. Another measuring tool to assess paracellular permeability, Dextran Blue (DB; molecular weight 2,000,000) was also used. After $72 \mathrm{~h}$ of incubation with media or media with IL-6, the monolayers were washed by media and then DB was administered into the apical chamber. After $2 \mathrm{~h}$ of incubation with DB, the media in the basal chamber was collected and measured using a spectrophotometer at $620 \mathrm{~nm}$. The DB concentrations were determined following comparisons with a standard curve of DB.

Human synovial SPLA 2 secreted into the media was measured by Enzyme Immunoassay (ELISA) kit (Cayman Chemical, Ann Arbor, Mich.). Media samples were collected, centrifuged, and the supernatants were stored at $-80^{\circ} \mathrm{C}$. All samples were analyzed according to the manufacturer's instructions. Briefly, $100 \mu \mathrm{l}$ of diluted samples were applied in duplicate to each well of a 96 well plate coated with a monoclonal antibody specific for $\mathrm{SPLA}_{2}$. Next, $100 \mu \mathrm{l}$ of acetylcholinesterase: Fab' Conjugate (AchE: Fab'), which binds selectively to a different epitope on the $\mathrm{SPLA}_{2}$ molecule, was added to the wells and incubated overnight at $4^{\circ} \mathrm{C}$. All solutions from the wells were removed, and the wells were rinsed six times with a wash buffer, followed by the addition of $200 \mu \mathrm{l}$ of Ellmen's Reagent and allowed to incubate $3 \mathrm{~h}$ in the dark at room temperature. The absorbance was read at $420 \mathrm{~nm}$ using model 550 Microplate Reader (Bio-Rad, Hercules, Calif.) and the results were compared with a standard curve of $\operatorname{sPLA}_{2}$.

Phospholipid composition of the media and cell lysates was measured after $72 \mathrm{~h}$ of incubation with media or media with IL-6. Four-milliliter media samples were collected and immediately frozen and stored at $-70^{\circ} \mathrm{C}$ until assayed. The cell monolayers were harvested by trypsinization with trypsin-EDTA and the cell lysate was immediately frozen and stored at $-70^{\circ} \mathrm{C}$ until assayed. Total lipids were extracted using a chloroform/methanol method [13]. Lipid extracts were dried under nitrogen gas, dissolved in chloroform and the total phospholipid content determined as phospholipid phosphorus [14, 15]. Extracted total phospholipids were separated by thin-layer chromatography (TLC) on silica gel 60 plates using chloroform/methanol/acetic acid/water (60:18:21:1.8). Plates with individual phospholipids were placed in $8 \%$ (wt./vol.) $\mathrm{CuSO}_{4}$ phentahydrate in water/methanol/concentration $\mathrm{H}_{3} \mathrm{PO}_{4}(60: 32: 8)$ or $0.1 \%$ (wt./vol.). Ninhydrin in ethanol was used for visualization after autoclaving $\left(150^{\circ} \mathrm{C}, 15 \mathrm{~min}\right.$, $110^{\circ} \mathrm{C}, 10 \mathrm{~min}$, respectively). The TLC plates were scanned and the individual PL's band images were then quantified using an imaging computer program Kodak EDAS 290 system (Kodak, Rochester, N.Y.). Individual phospholipid concentrations were compared with the following phospholipids; egg-yolk PC or lysophospholipid; LPC (Sigma) which functioned as standards. The percent of phosphatidylcholine from the chromatographic analysis was used to calculate the total PC content in the lipid extract. The results are reported as phosphatidylcholine phosphorus/milliliters of media and phosphatidylcholine phosphorus total amount of phosphate (mol.) of cells obtained from the epithelial monolayer.

\section{Statistical analysis}

Results are expressed as mean \pm SD. Statistical analysis was performed using linear regression analysis and one-way analysis of variance, with $P<0.05$ considered significant.

\section{Results}

The cytokine IL-6 caused a significant decrease in

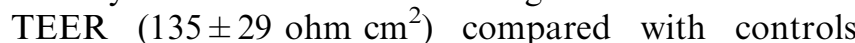
$\left(337 \pm 0.1 \mathrm{ohm} \mathrm{cm} \mathrm{cm}^{2} ; P<0.05\right.$; Fig. 1$)$.

IL-6 also caused a significant increase in Ma-Pm at both room temperature and at $4{ }^{\circ} \mathrm{C}(1.23 \pm 0.27$ and $0.50 \pm 0.06 \%$, respectively) compared with controls $(0.42 \pm 0.04$ and $0.14 \pm 0.03 \%$, respectively; $P<0.05$; Fig. 2); however, DB permeability following IL-6 administration did not change compared with controls.

Secretion of $\mathrm{sPLA}_{2}$ is shown in Fig. 3. IL-6 led to a significant increase in total $\mathrm{SPLA}_{2}$ secretion (143.8 \pm 12 ng sPLA $2 /$ mg DNA) compared with controls $\left(34.4 \pm 2.8 \mathrm{ng} \mathrm{sPLA}_{2} / \mathrm{mg} \mathrm{DNA} ; P<0.05\right)$.

There were no differences in total media PL composition between the two groups $(p>0.1)$. Total intracellular PL contents were also unchanged; however, percent ratios of specific PLs were altered after IL-6 treatment (Fig. 4). IL-6 led to significant changes in PL composition, including an increase in PE (9.6\%) and SM (5.7\%) and a decrease in PC $(8.7 \%)$ and LPC $(40.7 \% ; P<0.05)$ compared with controls. 


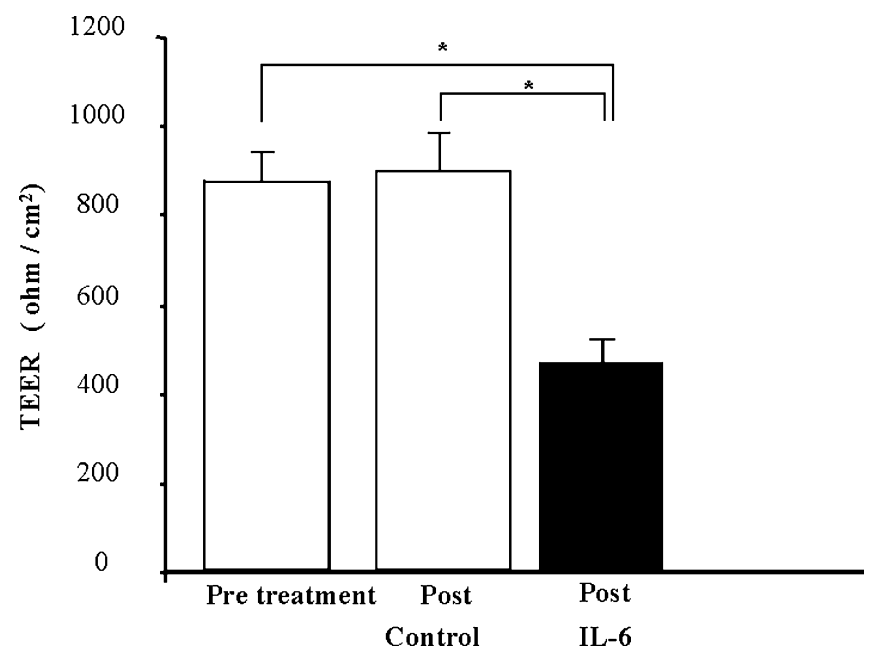

Fig. 1 Alteration of transepithelial electrical resistance (TEER) between pre- and post-treatment with media (control group) or media with IL-6 (IL-6 group). Asterisk Significant difference between controls and IL- 6 treatment group, $P<0.05$. Open columns control group, solid column IL-6 group

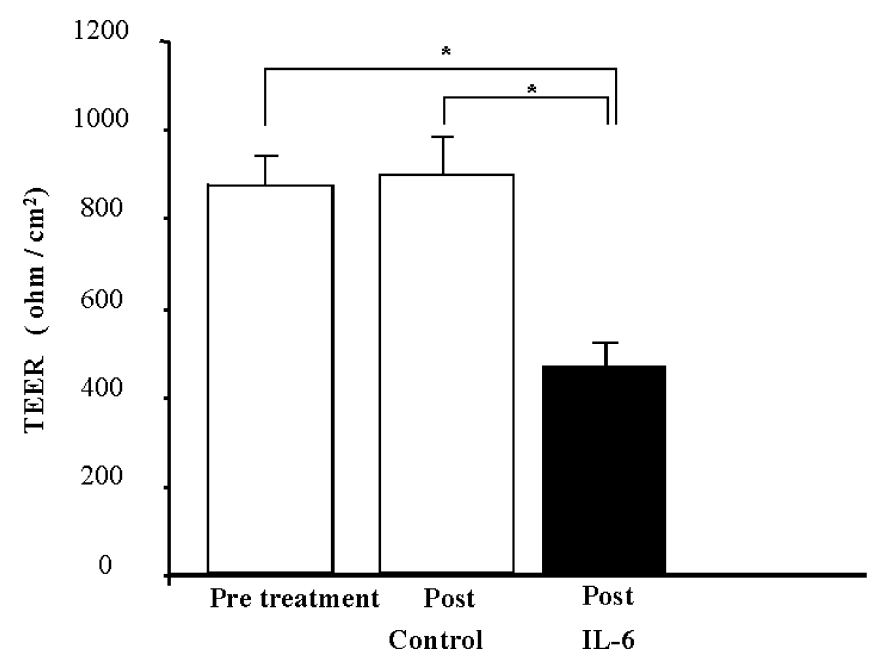

Fig. 2 Effect of IL-6 treatment on ${ }^{3} \mathrm{H}$-mannitol permeability at room temperature and at $4^{\circ} \mathrm{C}$. Asterisk Significant difference between controls and IL- 6 treatment group, $P<0.05$. Open columns control group, solid column IL-6 group

\section{Discussion}

Cytokines, specifically IL-6, have been shown to be increased in patients with IBD $[1,2,3,16]$. Moreover, IBD patients are more prone to develop bacterial translocation (BT), which may be related to increased intestinal epithelial permeability. Phospholipases have been recognized as a major enzyme group which have a critical role in diverse cellular responses, including phospholipid digestion and metabolism, host defense and signal transduction [17]. Secretory $\mathrm{PLA}_{2}$ and cytosolic $\mathrm{PLA}_{2}$ $\left(\mathrm{cPLA}_{2}\right)$ have different functions; $\mathrm{sPLA}_{2}$ appears to be a key factor in the inflammatory process [17], whereas $\mathrm{cPLA}_{2}$ functions mainly as a cell signaling factor [18].

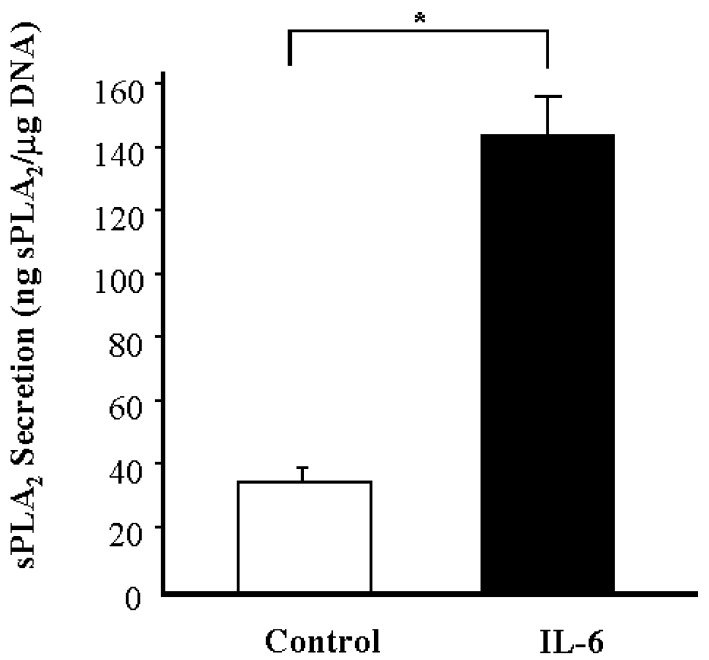

Fig. 3 sPLA 2 secretion after $72 \mathrm{~h}$ of IL- 6 treatment measured by ELISA. Asterisk Significant difference between controls and IL-6 treatment group, $P<0.05$. Open column control group, solid column IL-6 group

Increased $\mathrm{sPLA}_{2}$ is found in inflammatory fluids as well as in the serum of patients with rheumatoid arthritis [19], septic shock [8, 9], and IBD [20, 21, 22]. The expression of $\mathrm{SPLA}_{2}$ is increased in a variety of mammalian tissues, and is also increased in cancer patients [23]. The $\mathrm{SLA}_{2}$ is understood to be correlated with cell growth, as demonstrated in an in vitro model of epithelial injury, in which $\mathrm{sPLA}_{2}$, in a dose-dependent manner, stimulated rat intestinal epithelial cells (IEC-6) to migrate [24]. Other studies in murine intestinal cells have shown that ${ }_{\text {SPLA }}$ inhibitors prevented the growth of malignant cells [25]. In our preliminary study, sPLA $_{2}$ secretion showed a linear rise $(r=0.89)$ with increasing cell numbers, which suggests that sPLA $_{2}$ may have a role in growth or stabilization of cells under normal conditions (presented at the 14th International Symposium on Pediatric Surgical Research, 2001, Madrid).

Cytokine administration shows a close correlation with SPLA $_{2}$ secretion and leads to a reduction in TEER. Recombinant human IL-6 can increase serum concentrations of $\mathrm{sPLA}_{2}$ in humans [26]. Crowl et al. showed that IL-6 led to an up-regulation of $\mathrm{sPLA}_{2}$ gene expression in human hepatoma cells [27].

We hypothesized at the initiation of this study that high levels of the cytokine IL-6, as seen in IBD, would stimulate the secretion of $\mathrm{sPLA}_{2}$, resulting in epithelial barrier function breakdown through the increased LPC produced by $\mathrm{SPLA}_{2}$. In our study, we showed that IL-6 led to both a decline in TEER and an increase in Ma-Pm, suggesting that epithelial barrier function is decreased. In addition, we showed increased $\mathrm{SPLA}_{2}$ secretion in the IL-6 treated epithelial cell culture model. Based on these findings, we hypothesized that the increased $\mathrm{SPLA}_{2}$ would correlate with increased LPC products or alteration of secreted PLs in the media; however, the levels of PLs secreted into the 
Fig. 4 Phospholipid (PL) alteration after IL-6 treatment measured by thin-layer chromatography. Asterisk controls and IL-6 treatment group, $P<0.05 . P C$ phosphatidylcholine, $P G$ phosphatidylglycerol, $P E$ phosphatidylethanolamine, $S M$ lysophosphatidylcholine. Open columns control group, solid columns IL-6 group Significant difference between sphingomyelin, $L P C$

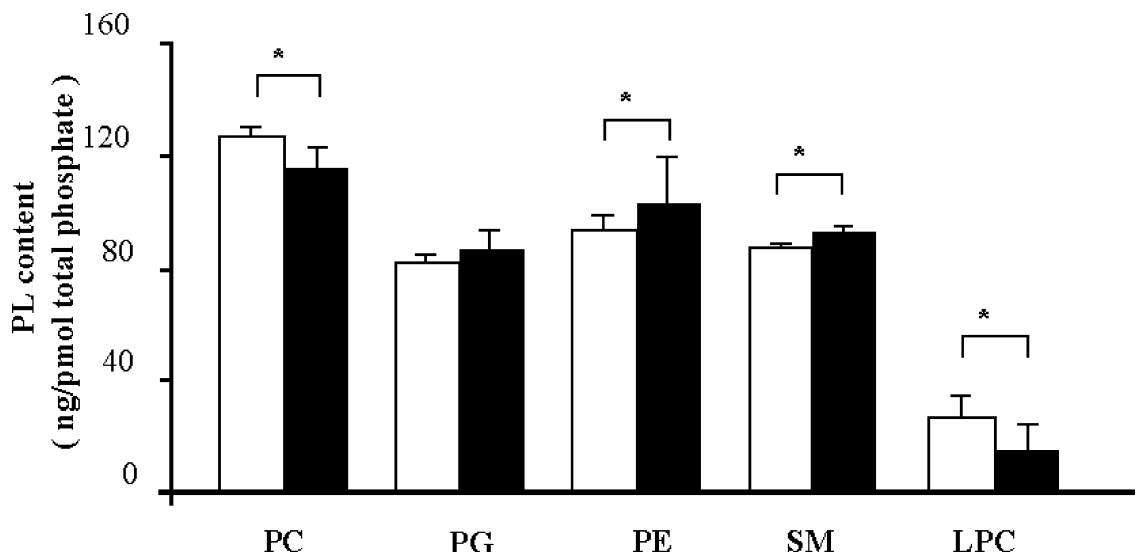

media were unchanged compared with controls. It is possible that secreted sPLA 2 as induced by IL-6 might have another action which induces the loss of epithelial membrane integrity. One such possibility is an alteration in the formation of intracellular lipid content, as it has been shown that $\mathrm{sPLA}_{2}$ can promote cholesterol absorption [28].

On the other hand, it was interesting to note that intracellular PLs were significantly altered following IL6 treatment in this model. These changes consisted of increased PE and SM and a decrease in PC and LPC. The PE and SM have been identified as signaling molecules in cellular apoptosis [29, 30, 31]. In our model, apoptotic changes were not evident at $72 \mathrm{~h}$ following Il-6 treatment (data not shown).

An increase in IL-6 caused by an inflammatory process in the intestine might have the following physiological effects: one is increased permeability caused by decreased epithelial cell membrane resistance and increased uptake of antigens through paracellular routes or by active transport. Secondly, increased IL-6 production may stimulate increased $\mathrm{sPLA}_{2}$ secretion into the intestinal lumen, which then leads to secondary epithelial membrane breakdown. Thirdly, increased IL-6 secretion may increase the intracellular PL's PE and SM which function as signaling compounds for either inflammation or apoptosis.

\section{Conclusion}

In conclusion, this study demonstrates that IL-6 mediates $\mathrm{SPLA}_{2}$ secretion and causes a decrease in epithelial integrity as measured by TEER and Ma-PM; however, the increased $\mathrm{sPLA}_{2}$ produced by IL- 6 treatment does not lead to increased LPC concentrations. On the other hand, IL-6 secretion leads to significant alterations in phospholipid content including intracellular phosphatidylethanolamine and sphingomyelin. It is possible that there may be a relationship between the increased $\mathrm{SPLA}_{2}$ produced by IL- 6 treatment and alteration in intracellular PL composition. Further investigation is needed, however, to better understand this mechanism. These facts might help us to understand the mechanisms involved in the loss of epithelial permeability in IBD patients and its relationship to increased cytokine production, especially IL-6.

\section{References}

1. Sartor RB (1994) Cytokines in intestinal inflammation: pathophysiological and clinical considerations. Gastroenterology 106:533-539

2. Sartor RB (1994) Cyclosporine therapy for inflammatory bowel disease. N Engl J Med 330:1897-1898

3. Reinecker HC, Steffen M, Witthoeft T, Pflueger I, Schreiber S, MacDermott RP, Raedler A (1993) Enhanced secretion of tumour necrosis factor-alpha, IL-6, and IL-1 beta by isolated lamina propria mononuclear cells from patients with ulcerative colitis and Crohn's disease. Clin Exp Immunol 94:174-181

4. Gennari R, Alexander JW, Pyles T, Hartmann S, Ogle CK (1994) Effects of antimurine interleukin-6 on bacterial translocation during gut-derived sepsis. Arch Surg 129:1191-1197

5. Kishikawa H, Miura S, Yoshida H, Hirokawa M, Nakamizo H, Higuchi H, Adachi M, Nakatsumi RC, Suzuki H, Saito H, Ishii H (2002) Transmural pressure induces IL-6 secretion by intestinal epithelial cells. Clin Exp Immunol 129:86-91

6. Molmenti EP, Ziambaras T, Perlmutter DH (1993) Evidence for an acute phase response in human intestinal epithelial cells. J Biol Chem 268:14116-14124

7. Ziambaras T, Rubin DC, Perlmutter DH (1996) Regulation of sucrase-isomaltase gene expression in human intestinal epithelial cells by inflammatory cytokines. J Biol Chem 271:12371242

8. Vadas P, Scott K, Smith G, Rajkovic I, Stefanski E, Schouten BD, Singh R, Pruzanski W (1992) Serum phospholipase A2 enzyme activity and immunoreactivity in a prospective analysis of patients with septic shock. Life Sci 50:807-811

9. Vadas P (1984) Elevated plasma phospholipase A2 levels: correlation with the hemodynamic and pulmonary changes in gram-negative septic shock. J Lab Clin Med 104:873-881

10. Otamiri T, Franzen L, Lindmark D, Tagesson C (1987) Increased phospholipase A2 and decreased lysophospholipase activity in the small intestinal mucosa after ischaemia and revascularisation. Gut 28:1445-1453

11. Tagesson C, Telemo E, Ekstrom G, Westrom B (1987) Development of phospholipase A2 and lysophosphatidylcholine metabolising enzyme activities in the neonatal rat intestine. Gut 28:822-828

12. Sawai T, Drongowski RA, Lampman RW, Coran AG, Harmon CM (2001) The effect of phospholipids and fatty acids on tight-junction permeability and bacterial translocation. Pediatr Surg Int 17:269-274 
13. Holch JLM, Sloane Stanley GH (1959) A simple method for the isolation and purification of total lipids from animal tissues. J Biochem 226:497-509

14. Weinhold PAVC (1965) Phospholipid metabolism in the liver and lung of rats during development. Biochim Biophys Acta 106:540-550

15. Abe A, Gregory S, Lee L, Killen PD, Brady RO, Kulkarni A, Shayman JA (2000) Reduction of globotriaosylceramide in Fabry disease mice by substrate deprivation. J Clin Invest 105:1563-1571

16. Brown KA, Back SJ, Ruchelli ED, Markowitz J, Mascarenhas M, Verma R, Piccoli DA, Baldassano RN (2002) Lamina propria and circulating interleukin-6 in newly diagnosed pediatric inflammatory bowel disease patients. Am J Gastroenterol 97:2603-2608

17. Murakami M, Kuwata H, Amakasu Y, Shimbara S, Nakatani Y, Atsumi G, Kudo I (1997) Prostaglandin E2 amplifies cytosolic phospholipase A2- and cyclooxygenase-2-dependent delayed prostaglandin E2 generation in mouse osteoblastic cells. Enhancement by secretory phospholipase A2. J Biol Chem 272:19891-19897

18. Balsinde J, Balboa MA, Li WH, Llopis J, Dennis EA (2000) Cellular regulation of cytosolic group IV phospholipase A2 by phosphatidylinositol bisphosphate levels. J Immunol 164:53985402

19. Pruzanski W, Keystone EC, Sternby B, Bombardier C, Snow KM, Vadas P (1988) Serum phospholipase A2 correlates with disease activity in rheumatoid arthritis. J Rheumatol 15:13511355

20. Minami T, Tojo H, Shinomura Y, Komatsubara T, Matsuzawa Y, Okamoto M (1993) Elevation of phospholipase A2 protein in sera of patients with Crohn's disease and ulcerative colitis. Am J Gastroenterol 88:1076-1080

21. Minami T, Tojo H, Shinomura Y, Matsuzawa Y, Okamoto M (1994) Increased group II phospholipase A2 in colonic mucosa of patients with Crohn's disease and ulcerative colitis. Gut 35:1593-1598
22. Minami T, Tojo H, Shinomura Y, Tarui S, Okamoto M (1992) Raised serum activity of phospholipase A2 immunochemically related to group II enzyme in inflammatory bowel disease: its correlation with disease activity of Crohn's disease and ulcerative colitis. Gut 33:914-921

23. Yamashita S, Yamashita J, Ogawa M (1994) Overexpression of group II phospholipase A2 in human breast cancer tissues is closely associated with their malignant potency. Br J Cancer 69:1166-1170

24. Minami T, Zushi S, Shinomura Y, Matsuzawa Y (1996) Phospholipase A2 stimulation of rat intestinal epithelial cell (IEC-6) migration. Am J Physiol 271:G664-G668

25. Longo WE, Grossmann EM, Erickson B, Panesar N, Mazuski JE, Kaminski DL (1999) The effect of phospholipase A2 inhibitors on proliferation and apoptosis of murine intestinal cells. J Surg Res 84:51-56

26. Bertsch T, Banks RE, Forbes MA, Aufenanger J, Storr M, Illingworth JM, Perren TJ, Selby PJ, Kattermann R (1996) Phospholipase A2 activity in serum is induced during treatment with recombinant human interleukin-6 in patients with cancer. Ann Clin Biochem 33:565-567

27. Crowl RM, Stoller TJ, Conroy RR, Stoner CR (1991) Induction of phospholipase A2 gene expression in human hepatoma cells by mediators of the acute phase response. J Biol Chem 266:2647-2651

28. Homan R, Hamelehle KL (1998) Phospholipase A2 relieves phosphatidylcholine inhibition of micellar cholesterol absorption and transport by human intestinal cell line Caco-2. J Lipid Res 39:1197-1209

29. Hannun YA (1994) The sphingomyelin cycle and the second messenger function of ceramide. J Biol Chem 269:3125-3128

30. Kolesnick R (2002) The therapeutic potential of modulating the ceramide/sphingomyelin pathway. J Clin Invest 110:3-8

31. Dobrowsky RT, Kolesnick RN (2001) Analysis of sphingomyelin and ceramide levels and the enzymes regulating their metabolism in response to cell stress. Methods Cell Biol $66: 135-165$ 